\title{
Die ökologische Stellung der Höhlen- und Spaltengewässer innerhalb der subterranaquatilen Lebensbereiche
}

\author{
Von Siegfried Husmann ${ }^{1}$ ) \\ Mit fünf Abbildungen im Text $\left.{ }^{2}\right)$
}

Es sind jetzt nahezu 75 Jahre her, als H. Garman (1882, p. 240) versuchte, die Herkunft der Fauna weiträumiger Gebirgshöhlen zu deuten, indem er erklärte, daß die Vorfahren der Höhlentiere, insbesondere die der nichtaquatischen, wahrscheinlich bereits an ein Leben in der Erde ,angepaßt" waren, ehe die Höhlen entstanden; Spalten und Risse in felsigem Gestein könnten als primäre Lebensstätten der Höhlenfauna angesehen werden. $\mathrm{Zu}$ der gleichen Schlußfolgerung kam auch der Höhlenforscher C. H. Eigenmann (1909, p. 13), der dann sogar den Biotop „Höhle" als eine Sammelstätte (,collecting ground“) derartiger Spaltenbesiedler bezeichnete, nachdem H. Krause (1898, p. 652) in recht deutlicher Weise seiner im Gegensatz dazu stehenden Meinung Ausdruck verliehen hatte: „Nun, wer's glaubt, mag ja auch bei dem Glauben selig werden können, daß die Höhlen gleichsam zum Tummelplatz und Elysium der Blinden aller Thierklassen erschaffen seien..."

Dreiviertel Jahrhundert sind inzwischen vergangen; doch eine allseits anerkannte Antwort auf die Frage nach Herkunft, Wesen und ökologischer Stellung der „Höhlenfauna“" konnte bis heute noch nicht gefunden werden.

Die obenerwähnte, von Garman (1892) vertretene Auffassung, „Höhlentiere" seien in Wirklichkeit Immigranten aus endogäischen

\footnotetext{
1) Limnologische Flußstation des Max-Planck-Institutes für Limnologie, 6407 Schlitz/Hessen (Westdeutschland).

$\left.{ }^{2}\right)$ Anfertigung nach Entwürfen des Verfassers: Uta Schnell, Schlitz/ Hessen.
} 
Mikrokavernen, fand neuerdings wieder einen namhaften Höhlen- und Grundwasserforscher als entschiedenen Verfechter: St. Karaman (1954, p. 216) betont, es gäbe überhaupt keine „Höhlenfauna“. „Die in Höhlen vorgefundenen Landtiere sind eigentlich Bewohner der kleinen, engen Felsspalten, gleich unterhalb der Erdoberfläche." Mit gleichem Nachdruck wird von Karaman auch die Existenz einer äquatilen ,Höhlenfauna" in Abrede gestellt. Diese in Höhlentümpeln lebenden blinden und pigmentlosen Wassertiere werden ökologisch als Elemente der eigentlichen Grundwasserfauna eingeordnet, die nur durch Überschwemmungen oder mit Tropfwasser aus ihren mikrokavernalen Biotopen in Höhlengewässer geraten.

Im jugoslawisch geschriebenen Hauptteil seiner Abhandlung kommt Karaman (zit. n. Hadzi, 1958, p. 472) sogar zu der Feststellung, daß nur ein „Glauben an eine Höhlenfauna" bestehe.

Diese Formulierung Karamans klingt wie eine echohafte Antwort auf den oben zitierten Vorwurf ,Glaube macht selig", mit dem Krause (1892) die ersten Verfechter der heute von Karaman vertretenen Meinung abkanzelte.

Die von diesem erfolgreichsten jugoslawischen Grundwasserforscher als ,Glaube“ abgetane Auffassung über das Wesen der „Höhlentiere“ entstand - nach Karaman - historisch, weil man zuerst in befahrbaren Höhlen diese augenlosen, bleichen Wassertiere vorfand, die dann eben als ,Höhlentiere" bezeichnet wurden; doch durch die Entdeckung der „Grundwasserfauna" - so behauptet Karaman des weiteren - sei die ,"Theorie der Höhlenfauna" zu Fall gebracht worden.

Die Gegenseite wendete hierzu ein: ,Unserer Meinung nach ist eine solche Auffassung unhaltbar, weil sie zu einseitig ist, und was noch wichtiger ist, weil sie nicht den Tatsachen entspricht" (J. Hadzi, 1958, p. 472).

Die hier sich zeigende Aktualität des Problems der ökologischen Position der „Höhle“ und der „Höhlenfauna" gibt Anlaß, vom heutigen Stand der Grundwasser-Limnologie aus die ökologische Stellung der Höhlengewässer innerhalb der übrigen subterranaquatilen Lebensbereiche eingehend $\mathrm{zu}$ beurteilen.

\section{II}

Die im Untergrund der Landschaften in nahezu allen Regionen der Erde in Lücken und Spalten und weiträumigen Höhlungen sich sammelnden Gewässer unterliegen alle der Wirkung zweier Naturgegebenheiten, deren Gegebensein in die an sich so sehr eigenständigen ver- 
schiedenen subterranaquatilen Biozonen zu einem ökologischen Ganzen verbindet.

Der eine dieser beiden zwischen den unterirdischen Lebensstätten vermittelnd wirkenden Faktoren ist die in allen Subterrangewässern herrschende vollkommene Dunkelheit; der andere wird in Gestalt der totalen oder doch recht weitgehenden Isolation dieser endogäischen Gewässer gegenüber Temperaturschwankungen der Atmosphäre wirksam.

Dieses überall in unterirdischen Gewässern ökologisch so gravierend in Erscheinung tretende Extremfaktoren-Nebeneinander gibt dem Ökologen durchaus die Berechtigung, die Gesamtheit aller endogäischen Süßwasserlebensbereiche als „Großlebensraum“ (,Ökoregnum") anzusehen und folgerichtig auch allgemein von einer „,Grundwasserfauna" zu sprechen: von ,,stygobionten“, ,,stygophilen“" und ,,stygoxenen“ Grundwassertieren (A. Thienemann, 1925, p. 32); gleichgültig, ob diese Subterranorganismen in grundwasserführenden Lückensystemen von Sanden, Kiesen oder Grobgeröllen leben, in Spaltengewässern oder in Höhlentümpeln.

\section{III}

Der von den verschiedensten unterirdischen Gewässern erfüllte Großlebensraum ,Grundwasser" - das Ökoregnum, ,Stygon“ (S. Husmann, 1966) - erstreckt sich von grundwasserführenden weiträumigen Gebirgshöhlen über Spaltensysteme in anstehendem Gestein und weiter in die Zwischenräume von Gehängeschutt sowie in Mikrokavernenlabyrinthe kiesiger und sandiger Lockergesteinsablagerungen. Alles in allem erfüllen also die von Grundwassertieren besiedelten unterirdischen Gewässer ein weitverzweigtes Hohlraumsystem verschiedensten Volumens, das von Fels oder von Lockergesteinen aufgebaut sein kann.

Die kavernale Geräumigkeit der grundwasserführenden Lebensstätten bietet naturgemäß jeweils nur morphologisch geeigneten Gestaltstypen der unterirdischen Wassertierwelt Einlaß und Bewegungsfreiheit. Daher können grundwasserführende Kavernen mit einheitlich geräumigem Volumen für morphologisch ,,passende "Grundwassertiere den ,eigentlichen" Biotop darstellen, wie es ja besonders eindrucksvoll für die ,Lebensformtypen“ (Remane) der „Sand"- und Kieslückenfauna" der Fall ist, während diese intergranularen, extrem engen Lückensysteme andererseits für Grundwassertiere mit ,,zu großem" Körperumfang zwangsläufig zu einer ,ökologischen Schranke" werden. 

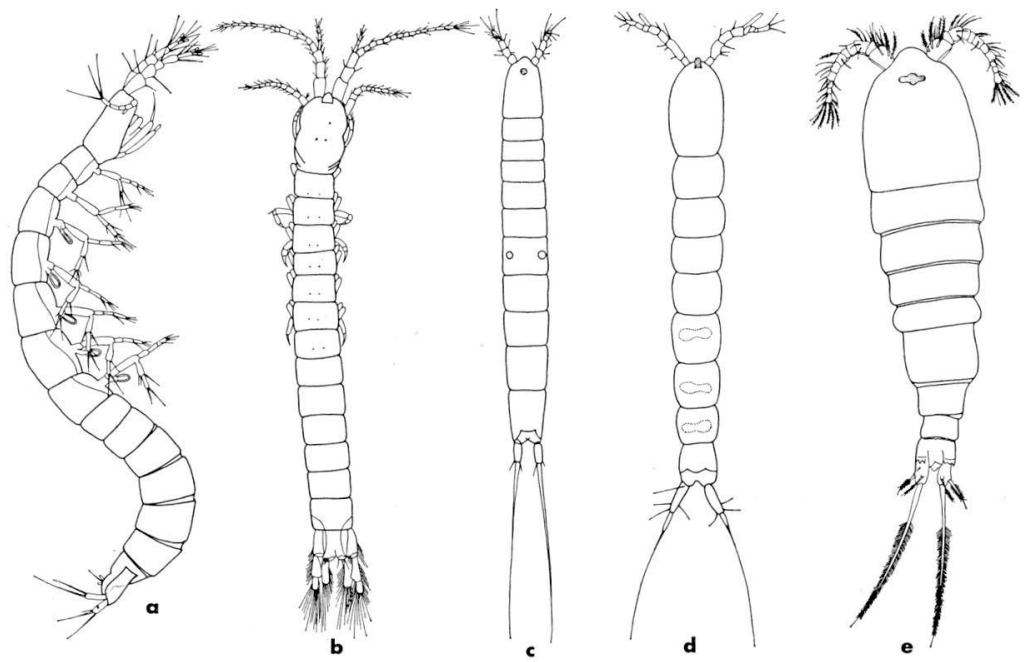

Abb. 1. Lebensformtypen des Mesopsammon; a: Leptobathynella amyxi (Siewing, 1956) (n. R. Siewing, 1956), b: Parastygocaris andina Noodt 1962 (n. W. Noodt, 1962), c: Horsiella brevicornis (Douve 1904) (n. W. Noodt, 1958), d: Parastenocaris entzi Török 1935 (n. P. Török 1935), e: Graeteriella unisetigera (Graeter 1908) (n. R. Gurney, 1932/1933).

So bietet sich der grundwassergefüllte Großlebensraum weitgehend als ein System ,ökologischer Nischen“ - in des Wortes treffendster Bedeutung - dar, die ihren typischen Besiedlern nur im ureigenen Bereich und zudem in geräumigere Hohlraumsysteme hinein Expansionsmöglichkeiten bieten.

Daher steht auch innerhalb der Fauna des grundwasserführenden Großlebensraumes ganz naturgemäß den Besiedlern von Sandlückensystemen das weiteste subterranökologische Feld zur Invasion offen; und tatsächlich gibt es einige Formen mesopsammaler Grundwassertiere, die offenbar in der ganzen Stufenfolge subterrankavernaler Grundwasserbiotope existenzfähig sind, wie beispielsweise Troglochaetus beranecki Delachaux sowie die Gattungen Parastenocaris Kessler (Abb. 1d), Graeteriella (Graeter) (Abb.1e) und Bathynella Vejdovsky, die eindeutig als typische Elemente des Mesopsammons gelten müssen, dabei aber die hier besonders hervorgehobene physiologische Bereitschaft ,,mitbringen“, auch in grundwasserführenden Bereichen von größerer Geräumigkeit gedeihen zu können.

Auch von einigen Elementen der ,Kieslückenfauna“ ist es bekannt, daß sie nicht allein in ihren „Hauptökotopen“ (Briegleb) vorkommen, 
sondern auch in Spaltengewässern und Höhlentümpeln; einige Beispiele: Asellus (Proasellus) casaticus Schiödte, Arten der Gattung Niphargus sowie Microniphargus leruthi Schellenberg.
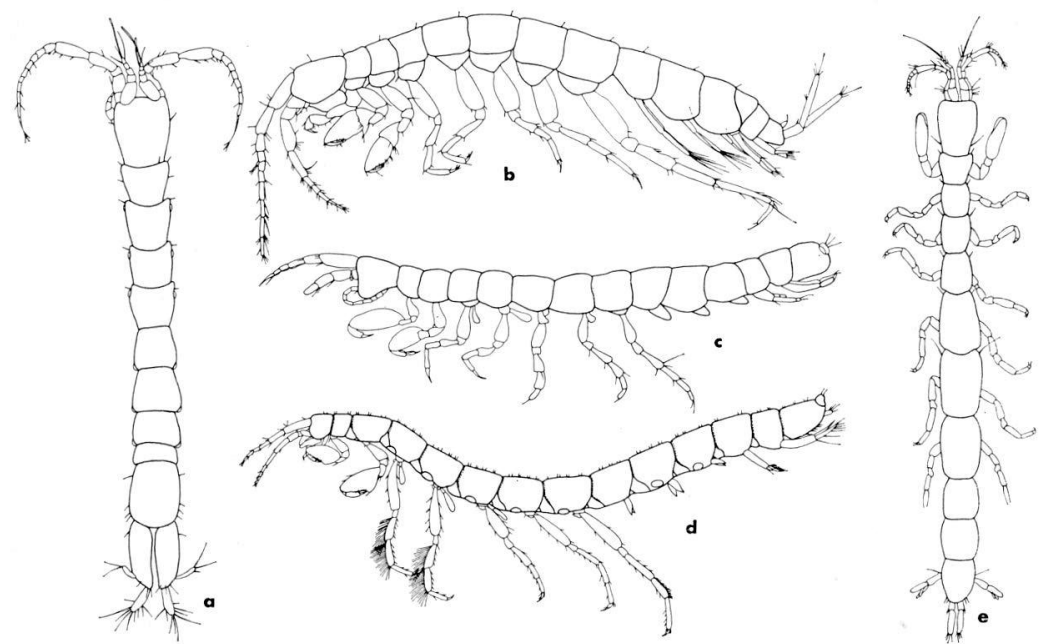

Abb. 2. Lebensformtypen des Mesopsephon; a: Microcharon stygius Karaman 1933 (n. St. Karaman, 1940), b: Bogidiella albertimagni Hertzog 1933 (n. St. Karaman, 1943), c: Ingolfiella acherontis (Karaman 1935) (n. St. Karaman, 1935), Ingolfiella leleupi Ruffo 1951 (n. S. Ruffo, 1951), e: Microcerberus stygius Karaman 1933 (n. St. Karaman, 1940).

Die durch ihre weite Geräumigkeit ausgezeichneten Felshöhlen mit den sich darin ansammelnden Wässern können bei einer dergestaltigen hohlraumabhängigen Besiedlungsstoßrichtung geradezu als ein besonders extremer Abschluß einer sich in allmählich größer werdender Raumausdehnung äußernden Stufenfolge von Subterranbiotopen angesehen werden.

Es liegt nahe, daß die weiträumigsten grundwasserführenden Hohlräume der Erdrinde wegen ihrer relativ leichten Zugänglichkeit den ersten Einblick in die unterirdische Wassertierwelt gestatteten (vgl. p. 410) und daß diese blinden, pigmentlosen, milchigweißen Besiedler der Höhlengewässer in ihrer Gesamtheit unvoreingenommen als Faunenelemente einer eigenständigen ,Höhlenfauna" betrachtet werden mußten, wie ja auch die zunächst als zönobiont anzusehen gewesenen ,Höhlen"tiere folgerichtig als ,troglobiont" (Racovitza, 1907) oder ,eucaval“" (Hesse, 1924) benannt wurden, wobei also eindeutig als Biotop zunächst nur die weiträumige Gebirgshöhle eine namensbestimmende Stellung einnahm, bis schließlich auch die weit- 
verzweigten Kleinsthöhlen - wie oben erwähnt - als Bioregionen in den Lebens,,raum" der ,,troglobionten“ - ,eucavalen“ - Tierwelt einbezogen werden mußten, und zwar sogar als ,eigentliche Biotope" einer ganzen Reihe dieser „Höhlentiere“ (vgl. p. 411).

\section{IV}

Es ist durchaus verständlich, daß nach der Entdeckung der artenund individuenreichen Interstitialfauna grundwasserführender Lockergesteine zunächst eine intensive Bestandsaufnahme der in diesen Lückenbiotopen lebenden Wassertiere einsetzte (vgl. Arbeiten von Angelier, Chappuis, Delamare-Deboutteville, Haine, Husmann, Jakobi, Karaman, Kiefer, Kulhavý, Meštrov, Motaş, Noodt, Orghidan, Pennak, Picard, Priesel-Dichtl, Ruffo, Schäfer, Schwoerbel, Viets, Wegelin u.a.); und immer noch ist diese zönologische Erschließung in vollem Gange, denn aus allen grundwasserführenden Klimazonen der Erde werden laufend neue Arten von in Sand- oder Kieslückensystemen lebenden Grundwassertieren gemeldet.

Das Wiederfinden von „Höhlentieren“ im Interstitialwasser von Lockergesteinen forderte schon gleich nach der Entdeckung dieser Mikrokavernenfauna dazu heraus, den offensichtlich ökologisch so ungeahnt vielgestaltigen Lebensraum dieser Elemente der „Höhlenfauna" in ökologische Bereiche eigener Prägung aufzugliedern.

Der Altmeister der biologischen Grundwasserforschung P. A. Chappuis war es, der den zunächst für das gesamte Grundwasser als Bezeichnung verwendeten Begriff ,nappe phréatique“ (vgl. Chappuis, 1950) schließlich zur speziellen Hervorhebung des in Lückensystemen von gerollten Lockergesteinen im Untergrund von Talauen und Terrassen ziehenden Interstitialwassers einführte (Chappuis, 1950), um diesen eigentlichen Lebensbereich der Sand- und Kieslückenfauna den grundwasserführenden Spalten und Höhlen gegenüberzustellen.

Die Begriffe ,nappe phréatique“ (Chappuis, 1950) oder ,phreatischer Biotop" (Motaş, 1958) betonen mit Recht die ökologische Einheit grundwasserführender Kavernensysteme in sandigen und kiesigen Aufschüttungen, ganz gleich, ob diese Lockergesteine den bodenbedeckten Untergrund von Talauen und Terrassen zusammensetzen oder aber Fließgewässerschotterbetten aufbauen.

Mit den Bezeichnungen ,ufernahes“" (S. Husmann, 1954, 1956) oder ,hyporheisches" (Orghidan, 1955) Grundwasser wurde der sandigkiesige Lückenbiotop in Gebirgsbachbetten als Lebensstätte eigener Prägung innerhalb des ,phreatischen Lebensraumes“ (Motaş) hervorgehoben und dem , interstitiellen Grundwasser in Talauen und Terrassen" (Husmann, 1954, 1956) gegenübergestellt. 
Bei der Abgrenzung dieser ökologischen Bereiche fanden jedoch die oben hervorgehobenen Befunde keine Berücksichtigung, daß nämlich „Sandlückensysteme“ und „,Kieslückensysteme“ als ,,Hauptökotope“ (Briegleb) ganz bestimmter, charakteristischer Lebensgemeinschaften anzusprechen sind. Um nun aber der ökologischen Bedeutsamkeit gerade der hohlraumformenden Substratkomponenten gerecht zu werden, war es notwendig, bei der Einteilung des interstitiellen Lockergesteinsgrundwassers in Lebensbereiche eigener Prägung neben der geomorphologischen Situation noch das Substratgefüge namensgebend heranzuziehen, mit anderen Worten: den "Sandlückenbiotop“" (,Psammal“", Sassuchin, Kabanov, Neizwestnova, 1927; „Mesopsammal“, Remane, 1940; „Limnopsammal“, Ruttner-Kollisko, 1957) und den Lebensraum der ,Kieslückenfauna“ (,Mesopsephal", Husmann, 1966) in entsprechender Weise zu kennzeichnen, das heißt: in Hinblick auf ihre Beziehungen zur Erdoberfläche oder aber hinsichtlich einer unter Umständen bestehenden totalen Isolation gegenüber Infiltrationen aus epigäischen Gewässern.

\section{V}

Die letztgenannten, endogäisch isolierten Biotope verdienen es auf Grund ihrer Abgeschlossenheit gegenüber biologischen Einflüssen aus oberirdischen Gewässern eigentlich einzig und allein mit Recht, daß man sie ohne jede Einschränkung nominell mit dem Strom der Unterwelt - „Styx“ - in Beziehung setzt (vgl. p. 411). Daher wurden wohlberechtigt derartige, gegenüber zönologischen Infiltrationen aus Oberflächengewässern abgeschirmte Bereiche des Großlebensraumes „Grundwasser" (,Stygon“) als „Eustygon“ (Husmann, 1966) besonders hervorgehoben und allen anderen Lebensbereichen des umfassenden ,Ökoregnums Stygon“ gegenübergestellt, die ihrerseits gerade dadurch zönologisch ausgezeichnet sind, daß ihre Lebensgemeinschaften sich aus Elementen teils echt subterraner, teils epigäischer Herkunft zusammensetzen, wie es beispielsweise für die in Lockerge steinsLückensystemen von Fließgewässerbetten lebenden Biozönosen innerhalb der Ökosysteme ,,Rhithron" und ,Potamon" (J. Illies, 1961) zutrifft. Die zönologisch vermittelnde Stellung dieser zwischen „Stygobios" einerseits und ,Rhithro"- beziehungsweise ,Potamobios" andererseits lebenden Biozönosen führte zu den Bezeichnungen „Stygorhithron" und „Stygopotamon" für die betreffenden grundwasserführenden Lückenbiotope in Fließgewässer-Schotterbetten (S. Husmann, 1966).

Die mesopsammale oder mesopsephale Beschaffenheit bestimmter Ökozonen dieser aquatilen Subterranbereiche wurde ebenfalls nomi- 
nell berücksichtigt, und zwar so, daß innerhalb des ,,phreatischen Biotops" (Motaş) folgende Lückenbiotope eigener Prägung gegenübergestellt wurden (S. Husmann, 1966):

$$
\begin{aligned}
& \text { „Eustygopsammal" - „Eustygopsephal" } \\
& \text { "Rhithrostygopsammal" - ,,Rhithrostygopsephal“" } \\
& \text { „Potamostygopsammal" - ,,Potamostygopsephal" }
\end{aligned}
$$

Über die ökologische Stellung dieser grundwasserführenden Interstitialbereiche der subterranen Biosphäre und hinsichtlich der zönologischen Eigenarten ihrer Lebensgemeinschaften wurde an anderer Stelle ausführlich berichtet (S. Husmann, 1966), so daß es hier genügen muß, im gesteckten Rahmen dieser Studie nur zusammenfassend das für unsere Erörterungen Wesentliche daraus zu skizzieren.
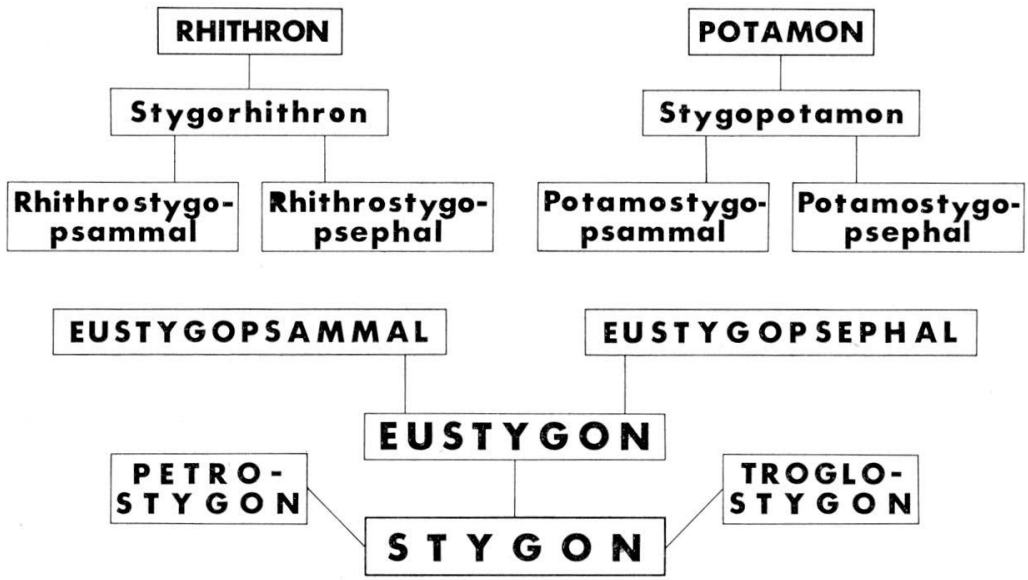

Abb. 3. Die Lebensbereiche des Ökoregnums ,,Stygon“.

(Aus: S. Husmann 1966)

Im Gegensatz zu den mesopsammalen und mesopsephalen Biotopen der Grundwasserfauna wurde für ,Spaltengewässer" im anstehenden Gestein sowie für aquatile Höhlenbiotope deren naturgegebene Zugehörigkeit zum ,Ökoregnum Stygon“ zunächst nur namensgebend hervorgehoben (Husmann, 1966, p. 244), indem für grundwasserführende Spalten in Felsgestein die Bezeichnung

$$
\text { ,.Petrostygon" }
$$

und für Grundwasseransammlungen in weiträumigen Gebirgshöhlen der Terminus

$$
\text { ,Troglostygon“" }
$$

vorgeschlagen wurde; und wenn nun die ökologische Stellung der Höhlen - und Spaltengewässer hier näher umrissen werden soll, so 
geschieht das also zugleich mit dem Ziel, die oben angedeutete Gliederung, die ja nur einen bestimmten Teil des Lebensraumes Grundwasser berücksichtigt, so zu erweitern, daß damit alle Lebensbereiche des Ökoregnums „,Stygon“ erfaßt werden.

\section{VI}

In der immer deutlicher hervortretenden ökologischen Erscheinung, daß die große Masse aquatiler , Höhlen“tiere als ,,eigentlichen Biotop" grundwasserführende Lückensysteme in Lockergesteinsaufschüttungen besiedelt (vgl. p. 411), sah St. Karaman (1954), wie schon berichtet wurde (vgl. p. 410), einen ausreichenden Anlaß, die, Theorie der Höhlenfauna" als ,zu Fall gebracht" anzusehen.

J. Hadzi (1958, p. 472) bezieht gegen die Auffassung, es gäbe in Wirklichkeit gar keine zönobionten Besiedler weiträumiger Gebirgshöhlen Stellung (vgl. p. 410) und betont, daß es eine Anzahl Tiere gibt, die ausschließlich in eigentlichen, begehbaren Höhlen gefunden wurden, und in Hinblick auf diese ökologische Gruppe könne man sich überhaupt nicht vorstellen, daß ihre Elemente als Glieder einer ,faune interstitielle" existieren könnten. Speziell an aquatilen Höhlentieren werden in diesem Zusammenhang allerdings nur die Garnelen (Decapoda/ Natantia) genannt, während A. Vandel (1964, p. 19) - mit gleicher ökologischer Wertung - die cavernicolen Crustaceen Caecosphaeroma, Monolistra, Sphaeromides, Troglocaris sowie die Vertebraten Amblyopsis, Caecobarbus, Anoptichthys und Proteus namhaft macht, ,,die gewiß niemals in ihrer Siedlungsgeschichte die interstitiellen Biotope besiedelt haben"; sie sind, so fährt Vandel fort, ,,direkt von den Gewässern der Erdoberfläche in die unterirdischen Flüsse und Seen eingewandert".

J. Hadzi (1958, p. 472) und A. Vandel (1964, p. 19) sprechen in ihren Erwiderungen gegen die von Karaman (1954, p. 216) geäußerte Ansicht, es existiere überhaupt keine eigentliche „Höhlen"fauna, lediglich von ,,interstitiellen Biotopen", die nicht als Lebensstätten für die obengenannten „Höhlentiere" denkbar seien. Ganz abgesehen davon, daß es meines Erachtens durchaus möglich erscheint, die Lückenbiotope in ausgesprochenen Grobgeröllen auch für einige dieser „,Höhlentiere“ als besiedelbar anzusehen, sei noch darauf hingewiesen, daß es neben weiträumigen Gebirgshöhlen und dem Interstitialbiotop der Geröll-Lückensysteme noch grundwasserführende Bereiche gibt, die durchaus als Lebensstätten eigener Prägung innerhalb des Ökoregnums ,,Stygon" angesehen werden müssen und als ,Hauptökotope" (Briegleb) bestimmter ,Höhlentiere“ gelten dürften: die grundwasser- 
führenden Felsspalten und ferner Zwischenraumsysteme in unterirdischen Ablagerungen zerfallener, ungerollter Felsgesteine. Dieser Lebensbereich wurde als ,Grundwasser in Spalten und Gehängeschutt" (S. Husmann, 1956, p. 152) erstmals besonders hervorgehoben und später als „Petrostygon" (S. Husmann, 1966) der aquatilen Bioregion weiträumiger Gebirgshöhlen an die Seite gestellt.

In der sich auf Grund einer verschiedenen Geräumigkeit ergebenden Stufenfolge von subterranaquatilen Lebensbereichen eigener Prägung (vgl. p. 412) kann die endopetrale wasserführende Dunkelregion ökologisch vermittelnd zwischen den Geröll-Lückenbiotop grober Lockergesteine - ,Mesopsephal" - und den subterranaquatilen Lebensbereich in befahrbaren weiträumigen Gebirgshöhlen gestellt werden.

\section{VII}

Die endopetrische Bioregion „Petrostyg on " ist wegen ihrer relativ geringen Geräumigkeit und der naturgegebenen massiven Isolation für limnologische Untersuchungen nicht ohne ungewöhnliche Schwierigkeiten direkt zugänglich; aber dennoch können die unterirdischen Gewässer von Felsspalten und endopetrischen Mikrokavernen auf indirekte Weise zönologisch erfaßt werden, und zwar dort, wo sie in weiträumige, befahrbare Höhlen beziehungsweise in die darin lokalisierten Höhlengewässer einmünden, oder auch an der Erdoberfläche, da, wo diese endopetralen Grundwässer quellenbildend zutage treten.

Es ist das Verdienst von W. Briegleb (1962), erkannt zu haben, daß sogar eines der Paradebeispiele (vgl. p. 417) zönobionter Faunenelemente der aquatilen Lebensgemeinschaften großräumiger Karsthöhlengewässer offensichtlich als typischer Besiedler der endopetrischen Ökoregion „Petrostygon“" angesehen werden muß: der Grottenolm (Proteus anguinus Laur.); denn nach den Befunden des obengenannten Verfassers stellte es sich eindeutig heraus, ,, daß der Schwerpunkt des Olm-Ökotops in derartigen unberührten Klüften liegt, in denen die Lebensbedingungen den Ansprüchen des Olms besser entsprechen als in den als Fundort bekannten Höhlen" (vgl. p. 425-427), was auch neuerdings von J. Hadzi (1965, p. 25) durch die Mitteilung der Beobachtung eingeräumt wird, daß nicht selten bei Sprengarbeiten, in deren Verlauf enge, mit Wasser gefüllte Spalten aufgeschlossen werden, Olme zutage gefördert werden, und zwar mit dem aus solchen freigelegten Klüften ausströmenden Wasser.

Planmäßige subterranlimnologische Untersuchungen in Zusammenhang mit Sprengarbeiten dürften überhaupt im Verbreitungsgebiet des Olmes zu noch tiefer greifenden Ergebnissen in dieser Hinsicht 
führen; denn zweifellos finden sich im Ausflußwasser von auf solche Weise aufgeschlossenen „Petrostygon“-Bereichen neben Proteus auch dessen ,Nährtiere " und sonstige Elemente der Spaltengewässerfauna, unter denen durchaus weitere Crustaceen-Arten aus der von Vandel (1964, p. 19) hervorgehobenen Gruppe (vgl. p. 417) ausschließlicher Bewohner von Höhlengewässern erwartet werden können.

Es erscheint nun ganz besonders aufschlußreich, auch andere „Höhlentiere" daraufhin zu untersuchen, inwieweit deren ökologische Verteilung dafür spricht, daß es sich bei diesen in weiträumigen, befahrbaren Höhlen gefundenen Arten in Wirklichkeit um Elemente der Mikrokavernenfauna des „Petrostygon“ handeln könnte.

Für eine solche ökologische Beurteilung nehmen aquatile Subterrantiere, die in Brunnen angetroffen wurden, eine sehr aussagekräftige Stellung ein, weil ja das in Trinkwasserbrunnen sich sammelnde Grundwasser durchweg den englumigen Zwischenraumsystemen in Lockergesteinen, Gehängeschutt und Felsspalten entstammt, weswegen schließlich die in Brunnen gefundenen Subterranorganismen sich im allgemeinen als Besiedler derartiger grundwasserführender Lückensysteme ausweisen oder doch zumindest bekunden, daß ,ihre " systematische Gruppe Mikrokavernensysteme zu besiedeln vermag.

\section{VIII}

Unter den von Hadzi und Vandel (vgl. p. 417) hervorgehobenen „Höhlentieren“, die von diesen Autoren als ausschließlich in Höhlen lebend bezeichnet werden, kommen „Höhlengarnelen" (Decapoda Natantia) und „Höhlenfische“ (Pisces) auch in Brunnen vor. Es soll nun im folgenden untersucht werden, inwieweit angenommen werden kann, daß ein Vorkommen derartiger blinder Tiere in Brunnen zu der Schlußfolgerung berechtigt, daß der „Hauptökotop“ dieser „Brunnenfische" und ,-garnelen" im petrostygonalen Grundwasser liegt und daß vom „Petrostygon" aus sowohl Brunnen als auch weiträumige Gebirgshöhlen von blinden Fischen und Garnelen besiedelt werden können. Hierzu soll von einer Aufstellung der bisher bekanntgewordenen Brunnenfundorte dieser Tiergruppen ausgegangen werden:

\section{A. Decapoda Natantia als Elemente der Brunnenfauna}

\section{ATYIDAE}

1. Troglocaris anophthalmus anophthalmus (Kollar 1848).

a) Brunnen nahe Monfalcone (Italien, Venezia Giulia); G. Müller, 1931, p. 207.

b) Brunnen nahe San Giovanni di Duino (Italien, Venezia Giulia); G. Müller, 1931, p. 206. 
c) Brunnen nahe Pulj (= Pola) (Jugoslawien, Istrien); G. Müller, 1931, p. 207.

2. Typhlatya monae Chace 1954.

Brunnen nahe El Molino, West-Indien, Mona Island;

F. A. Chace, 1954, p. 319.

3. Stygiocaris lancifera Holthuis 1960.

a) ,"Kudumurra-Brunnen".

b) ,Milyering-Brunnen“.

c) Mehrere Brunnen nahe Milyering.

a, b, c) West-Australien, Nordwestkap-Halbinsel, Yardie Creek Station.

L. B. Holthuis, 1960, p. 48; G. F. Mees, 1962, p. 30.

4. Stygiocaris stylifera Holthuis 1960.

a) ,,Kudumurra-Brunnen“.

b) Mehrere Brunnen nahe Milyering.

a, b) West-Australien, Nordwestkap-Halbinsel, Yardie Creek Station.

L. B. Holthuis, 1960, p. 54; G. F. Mees, 1962, p. 30.

\section{PALAEMONID A E}

5. Palaemonetes (Alaocaris) antrorum Benedict 1896. Artesischer Brunnen bei San Marcos, Texas;

J. E. Benedict, 1896, p. 615.

6. Troglocubanus gibarensis (Chace 1943).

Brunnen; Cuba.

F. A. Chace, 1943, p. 28.

7. Typhlocaris galilea Calman 1909.

Zisterne der Wassermühle nahe Tabghah am Nordende des Galiläa-Sees;

A. Craig-Bennett, 1938, p. 797.

8. Macrobrachium faustinum (De Saussure 1857).

Brunnen, Pos Caranja, W.-Lima, Bonaire;

F. A. Chace \& L. B. Holthuis, 1948, p. 23.

9. Macrobrachium aff. faustinum (De Saussure 1857).

Brunnen, Pos Caranja, W.-Lima, Bonaire;

W. L. Schmitt, 1936, p. 372; L. B. Holthuis, 1956, p. 67.

10. Euryrhynchus wrzesniowskii Miers 1877.

Brunnen in Cayenne, Französisch Guyana;

E. J. Miers, 1877, p. 663.

11. Euryrhynchus burchelli Calman 1907.

Brunnen in Para, Brasilien;

W. T. Calman, 1907, p. 297.

\section{B. Fische (Pisces Teleostei) als Elemente der Brunnenfauna}

\section{GYPRINIDAE}

1. Eilichthys microphthalmus Pellegrin 1929.

Mehrere Brunnen in Eil, Ital. Somaliland;

J. Pellegrin, 1929, p. 364 . 
II. BAGRIDAE

2. Trogloglanis pattersoni Eigenmann 1919. Brunnen in San Antonio, Texas;

C. H. Eigenmann, 1919, p. 397.

3. Satan eurystomus Hubbs \& Bailey 1947.

Artesischer Brunnen nahe San Antonio, Texas;

C. L. Hubbs \& M. Bailey 1947, p. 8.

4. Prietella phreatophila Caranza 1954.

Brunnen in El Potrero, Coahuila, Mexico;

J. Carranza, 1954, p. 129 u. 136.

III. CLARIIDAE

5. Uegitglanis zammaranoi Gianferrari 1923.

Mehrere Brunnen bei Uegit, Ital. Somaliland;

L. Gianferrari, 1923, p. 1-3.

6. Horaglanis krishnai Menon 1950.

Ein Brunnen bei Kottayan, Kerala, Süd-Indien;

A. G. Menon, 1950, p. 66.

IV. SYMBRANCHIDAE

7. Anommatophasma candidum Mees 1962.

a) ,"Tantabiddi-Brunnen“.

b) ,Milyering-Brunnen“.

c) ,Kudumurra-Brunnen“.

a, b, c) Yardie Creek Station, Nordwestkap-Halbinsel, Australien.

G. F. Mees, 1962, p. 28.

\section{PYGIDIIDAE}

8. Phreatobius cisternarum Goeldi 1904.

Zisterne auf der Insel Marajo, Amazonas-Mündung;

C. R. Goeldi, 1904, p. 549.

VI. AMBLYOPSID A E

9. Chologaster agassizi Putnam 1872

(= Chologaster papilliferus Forbes 1872).

Brunnen in USA;

A. Vandel, 1964, p. 267.

10. Typhlichthys subterraneus Girard 1859

(= Typhlichthys wyandotte Eigenmann 1905).

a) Brunnen nahe Bowling Green Ky, Californien;

Ch. Girard, 1859, p. 62.

b) Brunnen nahe Corydon, Indiana;

C. H. Eigenmann, 1905, p. 63.

11. Amblyopsis spelaea De Kay 1842.

Brunnen in Indiana;

L. P. Woods \& F. Inger, 1957, p. 245.

\section{ELEOTRIDAE}

12. Milyeringa veritas Whitley 1945.

a) ,Milyering-Brunnen".

b) ,Kudumurra-Brunnen“.

a, b) Yardie Creek-Station, Nordwestkap-Halbinsel, Australien; G. F. Mees, 1962, p. 29. 
VIII. GOBIIDAE

13. Luciogobius pallidus Regan 1940.

Brunnen in Japan;

T. Regan, 1940.

Bei der Untersuchung obiger Brunnenbiotope von ,Höhlentieren“ fanden die grundwasserführenden Schichten, in die die betreffenden Grundwassersammelbehälter eintauchen, nur in wenigen Fällen besondere Beachtung. Dennoch gibt es einige für unsere Fragestellung besonders aufschlußreiche Angaben, die durchaus dafür sprechen, daß wassererfüllte Spaltensysteme und Zwischenraumlabyrinthe eindeutig als ,Hauptökotope“ (Briegleb) der in den genannten Brunnen angetroffenen durchweg blinden und pigmentlosen Garnelen und Fische angesehen werden müssen.

Besonders eindrucksvoll sind in dieser Hinsicht die von G. F. Mees (1962) beschriebenen Biotopverhältnisse innerhalb des grundwasserführenden Gesteins im Einzugsmantel der ,Garnelen- und Fischbrunnen" Westaustraliens, in denen zwei blinde Decapoda Natantia (vgl. p. 420):

Stygiocaris lancifera Holthuis 1960,

Stygiocaris stylifera Holthuis 1960,

angetroffen werden konnten und in denen außerdem noch zwei Arten augenloser Fische (vgl. p. 421) leben:

Anommatophasma candidum Mees 1962,

Milyeringa veritas Whitley 1945.

Nach G. F. Mees (1962, p. 24, 26) stehen die von diesen Arten besiedelten Brunnen in einer Küstenterrasse von $1 \frac{1}{2}-3 \mathrm{~km}$ Breite, deren Untergrund aus Korallengestein, Schnecken- und Muschelschalen aufgebaut ist. Diese Ablagerungen sind von Rissen, engen Höhlungen und verbindenden Gängen (,,crevices, small holes and connecting corridors") durchzogen, so daß ein ausgedehntes subterranes Netzwerk von Wasseradern (,an extensive subterranean network of waterways") existiert, ,which forms the habitat of the fauna under discussion". Zu dieser Feststellung, daß die grundwasserführenden Risse, Spalten und sonstigen Lückensysteme den speziellen Biotop dieser blinden Besiedler unterirdischer Gewässer darstellen, führte auch die Beobachtung, daß Milyeringa veritas durch Spalten und Risse der Brunnenwandung hindurch in den jeweils als Sekundärbiotop angenommenen Brunnen einwandert und diese Öffnungen auch zur Rückkehr ins anstehende „Lückengestein“ benutzt (G. F. Mees, 1962, p. 26).

Ein ähnliches Verhalten beobachtete G. Thines (1958, p. 120) bei Uegitglanis zammaranoi Gianferrari in einem Brunnen bei Uegit in Italienisch-Somaliland (vgl. p. 421): Die Tiere verließen hastig einen 
kleinen Spalt (,,une petite crevasse ${ }^{6)}$ zwischen den Steinen der Seitenwand, was darauf schließen will, daß Uegitglanis zammaranoi in Wirklichkeit ein Besiedler des Grundwasserstromes ist, in dem der untersuchte Brunnen steht, welch letzterer somit als ein mehr oder weniger zufällig aufgesuchter Lebensbereich (,un habitat accidentel“) anzusehen ist.

Auch Anommatophasma candidum Mees 1962 ist ein Besiedler von Spalten- und Lückensystemen, die mit dem von G. F. Mees (1962) untersuchten westaustralischen Brunnen in Verbindung stehen. Doch diese Art erreicht ihre Brunnenbiotope nicht aus frei von Grundwasser durchströmten Mikrokavernen. Die aalartige Gestalt dieses zur Familie Synbranchidae zählenden Brunnenbesiedlers verleiht diesem auffallend weiß aussehenden „Blindfisch“ die Fähigkeit, normalerweise sogar Gesteinsspalten zu besiedeln, die nicht nur von Grundwasser durchflossen, sondern zudem noch von organischen und anorganischen Zerfallsteilen (,mud and debris“") ausgefüllt sind (vgl. G. F. Mees, 1962, p. 24). Diese Subterranfische bewegen sich schlängelnd und wühlend durch die labyrinthartigen grundwasserführenden Spaltensysteme hindurch und erreichen dabei unter Umständen Brunnen als zusätzliche Lebensstätten ${ }^{1}$ ).

Neben diesen ,Brunnenfischen“ sind noch einige blinde Fischarten bekanntgeworden, die innerhalb von Flußbetten in Gesteinszwischenräumen leben. Sie deuten ebenfalls darauf hin, daß naturgegebene Mikrokavernen als Hauptökotope blinder Wassertiere angesehen werden müssen.

\section{IX}

Aus der Familie Pygidiidae begegnete uns in der vorstehenden $\mathrm{Zu}$ sammenstellung von "Brunnenfischen" bereits eine blinde Art Phreatobius cisternarum Goeldi 1904 -, deren Vorkommen in einem Brunnen auf der Amazonasinsel Marajo (vgl. p. 421) darauf schließen ließ, daß diese Art die Lückensysteme des Brunneneinzugsgebietes besiedelt.

Aus dem Stromgebiet des Amazonas wurden noch zwei weitere Arten von ,Blindfischen“ bekannt, die ebenfalls den Pygidiidae angehören, und zwar einer neuen Unterfamilie: Glanapteryginae Myers

1) Mit der Erwähnung der Möglichkeit einer derartigen ,,Untergrundpassage" (,,underground passage“) versucht bereits C. L. Hubbs (1936, p. 166) das sympatrische Vorkommen zweier Formen des Höhlenfisches Rhamdia guatemalensis zu deuten: "These two forms probably migrated into this cave by different underground passages", wie ja auch schließlich das Vorkommen zweier Stygiocaris-Arten in einem Brunnen Australiens (vgl. p. 420) nur durch Untergrundpassage von zwei Entwicklungszentren her erklärbar ist. 
1944. Sie wurden im Gesteinsbett des Rio Negro unterhalb der Stromschnellen von Sao Gabriel entdeckt (G. S. Myers, 1944, p. 593). Die langgestreckte Gestalt dieser in wassererfüllten Lückensystemen zwischen Steinen oder in Spalten lebenden Fischarten

Pygidianops eigenmanni Myers 1944,

Typhlobelus ternetzi Myers 1944

darf ebenso berechtigt als ,lebensformtypisch" bezeichnet werden, wie die der in Sand- oder Kieslückensystemen vorkommenden wurmförmig gestreckten biotopeigenen Arten (vgl. p. 411 u. Abb. 1, 2).

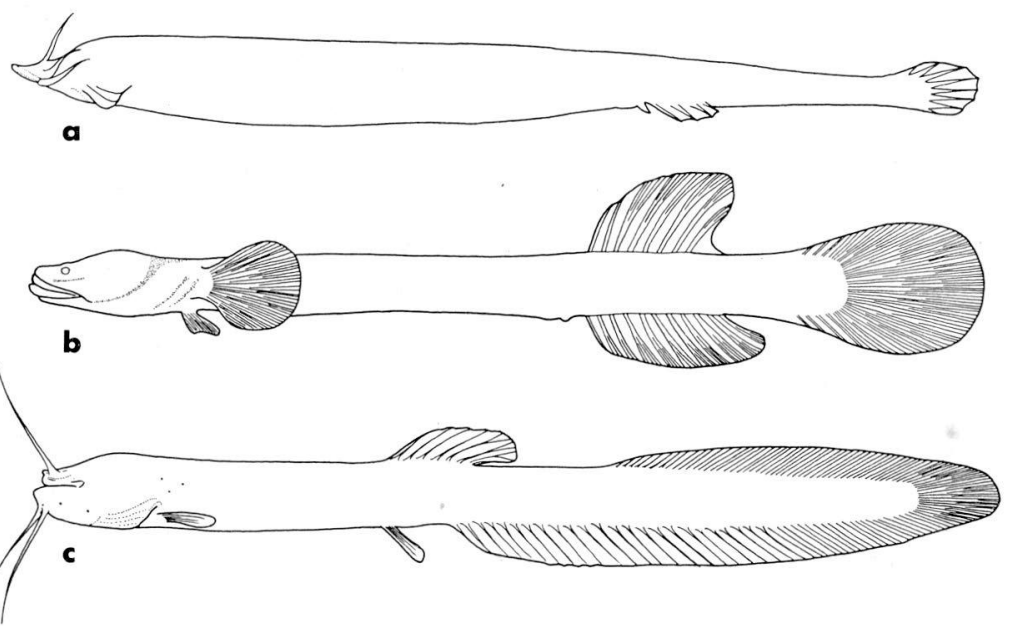

Abb. 4. Fische (Pisces) als Lebensformtypen der Lückensystemfauna; a: Typhlobelus ternetzi Myers 1944 (n. G. S. Myers, 1944), b: Luciogobius guttatus ama (Snyder 1909) (n. Snyder, 1909), c: Phreatobius cisternarum Goeldi 1904 (n.M.Reichel, 1927) (Längenmaße; a: 33,5 mm, b: 40,5 mm, c: 40,5 mm).

Aber auch die Blindheit und Pigmentlosigkeit der obengenannten „Rio-Negro-Fische“"1) stellt diese Arten - so wie die vorstehend erwähnten blinden, milchigweißen „Brunnenfische“ - ökologisch an die Seite von Subterranorganismen, deren Hauptökotop in wasserführenden Mikrokavernen zu suchen ist.

Blindheit und Pigmentlosigkeit - verbunden mit lebensformtypischer Gestalt oder relativ geringer Körpergröße - sind offenbar Eigenschaftskombinationen, die darauf schließen lassen dürfen, daß derartige Organismen als Hauptökotope Spaltengewässer oder sonstige wasserführende Lückenbiotope besiedeln.

1) Pygidianops eigenmanni ist vollkommen augenlos, Typhlobelus ternetzi weist Reste verkümmerter Augen auf; beide Formen sind vollkommen pigmentlos (G. S. Myers, 1944, p. 592, 593). 


\section{$\mathbf{X}$}

Die Annahme, daß wasserführende Zwischenraumsysteme im allgemeinen als ,eigentliche Biotope" blinder, pigmentloser Wassertiere anzusehen sind, da $\beta$ somit derartige in Brunnen, Höhlen oder Quellen angetroffene Faunenelemente zönologisch der Mikrokavernentierwelt zuzuordnen sind, diese ökologische Schlußfolgerung also ergibt sich auch aus einer naturgegebenen Regelmäßigkeit, nach der offensichtlich die Verteilung der Grundwassertiere innerhalb der subterranaquatilen Biotope eigener Prägung gesteuert wird. Für unser Vorhaben, die ökologische Position der Gewässer weiträumiger Gebirgshöhlen und ihrer Fauna festzulegen, ist es besonders aufschlußreich, den Lebens,,raum" „Höhle" in seiner Abhängigkeit von dieser subterranökologischen Gesetzmäßigkeit zu erfassen.

Das hier hervorgehobene allgemein in subterranlimnischen Biotopen wirkende ökologische Grundprinzip gibt sich vor allem in der Erscheinung zu erkennen, daß die Körpergestalt der Grundwassertiere - insbesondere der maximale Körperquerschnitt - insofern von entscheidender Bedeutung für die Hauptökotopwahl ist, als anscheinend grundsätzlich ein möglichst engräumiger grundwasserführender „Kleinsthöhlenbiotop" bevorzugt wird, dessen mikrokavernale Beengtheit allen ,zu großen" Feindarten zwangsläufig das Eindringen erwehrt. So leben ja auch bekanntlich die bei ihrem so überaus geringen Körperumfang extrem langgestreckten "Lebensformtypen“" (Remane) der "Sandlückenfauna“ (vgl. Abb.1) gemeinsam mit ihrer durch ,angemessene“ Körpergröße ausgezeichneten Begleitfauna in hochgradiger ,zönologischer Isolation“" gegenüber räuberischen Elementen der "Kieslückenfauna“. Diese Besiedler mesopsephaler Lückenbiotope finden in den Zwischenraumsystemen fein- bis grobkörniger Kiese ,ihren" optimalen Lebensbereich, und zwar ebenfalls auf Grund der auch darin gegebenen ökologischen Abgeschlossenheit gegenüber eigenen Feindarten von überlegener Körpergestalt. Auch die ,petrostygonalen“" Zwischenraumsysteme (vgl. p. 416) können ebenfalls entsprechende ökologische Schutzbereiche darstellen, was sich besonders deutlich bei den schon erwähnten (vgl. p. 418) speologischen Befunden von W. Briegleb (1962) zeigte. Diese allerdings in erster Linie die Ökologie des Olmes behandelnden Untersuchungsergebnisse enthalten Angaben, deren Auswertung im Rahmen unserer allgemein-subterranökologischen Fragestellung bedeutsame Aufschlüsse gibt; und daher soll noch einmal - entsprechend gezielt und schlußfolgernd - darauf eingegangen werden.

W. Briegleb (1962, p. 273, 274) stellt fest, daß die Olme mit ihrer stygobionten Begleitfauna innerhalb des untersuchten Höhlengewäs- 
sers nur in einem sogenannten „Randökotop“ leben, das heißt: in jener Höhlengewässerzone, in die das ,,petrostygonale" Grundwasser (vgl. p. 416) - aus dem eigentlichen ,Olm-Biotop" kommend - einmündet.

In diesem beengten „,Randökotop“ des Olmes fanden sich neben Proteus anguinus Laurenti noch andere, ,echte" Subterranorganismen: die Garnele Troglocaris spec. sowie Niphargus spec., die sich beide als wichtigste Beutetiere des Olmes erwiesen, ferner noch Asellus aquaticus (Linnaeus), mit Anpassungen an das Höhlenleben, sowie Trikladen.

Die außer Asellus aquaticus von der Erdoberfläche her in das von Briegleb untersuchte Höhlengewässer eindringenden ,zönophilen“ beziehungsweise ,zönoxenen“" „Höhlentiere“ - Forellen (Trutta), Flußkrebse (Astacus), Flohkrebse (Gammarus pulex), Trichopterenund Plecopterenlarven - überschreiten bezeichnenderweise eine bestimmte Grenze in der Tiefe des Höhlengewässers nicht; und diese „,vordere Linie" ihres sich durch den Höhleneingang in die Höhle einschiebenden Siedlungsgebietes ist zugleich die äußere Grenze des von Proteus, Troglocaris, Niphargus, Asellus und Trikladen besiedelten „Olm-Randökotops". Diese stygobionte Fauna findet im Bereich ihres Höhlengewässer-Refugiums eine offenbar sichere Zuflucht vor den gefräßigen Forellen (Trutta) und Flußkrebsen (Astacus), von denen diese Elemente der Spaltengewässerfauna buchstäblich ,,in die Enge getrieben" werden.

Die Tatsache, daß es den außerordentlich räuberischen Flußkrebsen und auch den sich ebenso verhaltenden Forellen ganz offensichtlich auf Grund ,innerer Faktoren" verwehrt ist, den Randökotop des Olmes (Proteus anguineus) und seiner stygobionten Begleitfauna über ein bestimmtes Maß einzuengen, zeigt, daß nicht immer nur allein der oben in den Vordergrund gestellte Grad der Geräumigkeit grundwasserführender Kavernen über das Eindringevermögen von außerhalb lebenden Tieren entscheidet, sondern daß die ,zönologische Isolation" kavernaler Biozönosen in bestimmten Fällen auch von Tieren nicht gestört werden kann, deren Körpergröße an sich ein Eindringen möglich machen würde, die aber innerhalb ihres ,Artbildes" nicht über die ,,physiologische Bereitschaft" verfügen, in unterirdischen Bereichen über eine bestimmte Grenze hinaus vorzudringen.

Diese Feststellung läßt erkennen, daß unser oben hervorgehobenes ökologisches Grundprinzip (vgl. p. 425) einer entsprechend umfassenderen Formulierung bedarf, zumal da sich im Wesen ähnliche zönologische Grenzbereiche auch in den Bioregionen der Mikrokavernenfauna nachweisen lassen, beispielsweise in den Übergangszonen vom „Stygorhithron“ zum „Eustygon“" (vgl. p. 415, 416), wo eine ganze 
Reihe von Arten der ,stygorhithrophilen“ Interstitialfauna nebst „,stygorhithrobionten“Wassermilben (Hydrachnellae und Halacaridae) es nicht vermag, über eine mehr oder weniger deutliche Grenze hinaus in die Randbereiche des Schotterbettes oder gar ins uferferne Talauengrundwasser (,Eustygon“) hinein vorzudringen (vgl.S. Husmann 1966).

Das für eine zönologische Beurteilung der ,Höhlenfauna "zweifellos sehr bedeutsame und auch innerhalb dieser Erörterungen daher verschiedentlich erwähnte ökologische Grundprinzip der Herausbildung von Hauptökotopen der Grundwasserfauna könnte bei Berücksichtigung der hier gekennzeichneten biotischen und abiotischen Faktoren in erster Fassung etwa so formuliert werden:

Der eigentliche Lebensbereich (Hauptökotop) von Grundwassertieren wird - neben sonstigen optimalen Lebensvoraussetzungen durch eine im Verhältnis zum Körperdurchmesser der einzelnen Arten extreme Engräumigkeit der besiedelten grundwasserführenden Kavernen gekennzeichnet, die deren Bewohnern eine hochgradige ,zönologische Isolation" und damit eine günstige ökologische Geborgenheit gewährt, indem allen ,,zu großen“ Feindarten und Nahrungskonkurrenten eine Immigration verwehrt wird. Diese ökologische Abschirmung gegenüber lästigen Mitbesiedlern kann zudem subterran stellenweise noch dadurch abgesichert werden, daß morphologisch an sich „,passende" Feindarten und Konkurrenten sich selber infolge einer ,physiologischen Ungeeignetheit" von der Immigration ausschließen.

\section{XI}

Wenn man nun also mit Recht für alle „Hauptökotope“ der Grundwasserfauna in bezug auf ihre charakteristischen Besiedler - neben allen sonstigen günstigen Lebensverhältnissen - eine auffallend extreme mikrokavernale Beengtheit als ganz besondere Voraussetzung der darin für diese Tiere herrschenden optimalen Lebensbedingungen ansehen kann, so müssen Gewässer in befahrbaren Höhlen als besonders ungünstige Lebens,,räume" stygobionter Grundwassertiere angesehen werden, weil darin der ökologisch so sehr bedeutsame Faktor ,Engräumigkeit" fortfällt. Das damit verbundene Fehlen einer existenzfördernden lebens,,raum"bedingten ,zönologischen Isolation“ (vgl. p. 425) kann sich in negativster Weise naturgemäß gegen typische Elemente der „Sand-“ und ,Kieslückenfauna“ auswirken, aber auch recht erheblich gegenüber Elementen der petrostygonalen (vgl. p. 416) Fauna, wie Briegleb (vgl. p. 426) herausfand.

Die Erfahrung, daß einzelne Arten der stygobionten Grundwassertierwelt unter Umständen Höhlengewässer recht individuenreich zu 
besiedeln vermögen, zeigt, wie sehr auch in Höhlen unter besonderen Voraussetzungen dennoch hin und wieder günstige Verhältnisse für Elemente der Lückenfauna herrschen können, beispielsweise dann, wenn sich diesen Tieren in Makrokavernen keine überlegenen räuberischen Wassertiere entgegenstellen, mit anderen Worten: wenn darin eine gleichwertig umfassende „ökologische Abgeschlossenheit“" herrscht, wie in den engräumigen Hauptökotopen der Interstitialfauna. Innerhalb von Höhlengewässern entscheidet also der Grad des zönologischen Einwirkens ,,lästiger" Faunenelemente über den Umfang einer biozönotischen Entfaltung dieser mikrokavernalen Grundwassertiere; und durch die hydrogeologische Verschiedenheit limnischer Höhlenbereiche wird es bedingt, daß es für die ökologischen Verhältnisse in „Wasserhöhlen“ ein ganzes Spektrum von Möglichkeiten gibt, von denen für Grundwassertiere jeweils eine zum Tragen kommen kann. Da diese Fülle ökologischer Möglichkeiten für in Höhlengewässer geratene Lückenbesiedler von einer günstigen ,zönologischen Isolation" bis zu einer totalen biozönotischen Unterlegenheit gegenüber Feindarten reichen kann, sind naturgemäß für Elemente der Interstitialfauna in Höhlengewässern alle möglichen Grade eines biozönotischen Sichbehauptens oder aber gar keine Existenzmöglichkeiten denkbar.

Die sich daraus ergebende Vielfalt biozönotischer Kombinationsmöglichkeiten spricht schon allein dafür, daß nicht nur die Berechtigung besteht, Höhlengewässer innerhalb des Ökoregnums „Stygon“ (vgl. p. 411) als Lebensbereiche besonderer Prägung anzuerkennen, sondern daß innerhalb dieser weiträumigen aquatilen Subterranregion sogar noch eine zönologische Zonierung vorgenommen werden kann, wie die von Briegleb (1962) erfaßten entsprechenden Verhältnisse in und an den Kontaktzonen stygobionter und rhithrobenthaler Biozönosen in Höhlen erkennen lassen (vgl. p. 426).

Ganz gleich, ob es überhaupt zönobionte Höhlentiere gibt oder nicht (vgl. p. 410), geschieht es also - so oder so - durchaus mit Recht, wenn von Grundwassertieren besiedelte Süßwasseransammlungen in Höhlen als aquatile Subterranbiotope eigener Prägung angesehen und als Bereiche eines besonderen Ökosystems - ,Troglostygon" - hervorgehoben werden.

\section{XII}

Der Lebensbereich der unterirdischen Gewässer in Höhlen, die Ökoregion ,Troglostygon “, umfaßt naturgemäß nur jene Höhlen, deren Gewässer eine rein limnische Natur aufweisen, ganz entsprechend der namensgebenden Beziehungsaufnahme zum kontinentalen 
Strom der Unterwelt „Styx“. Es gibt nun aber neben einem solchen „Limnotroglon" (=, Stygotroglon") noch einen weiteren makrokavernalen Lebensbereich eigener Prägung, dessen ökologische Eigenständigkeit durch einen über einer bestimmten Höhe liegenden Gehalt an im Wasser gelösten Salzen gekennzeichnet ist. Die ökologische Besonderheit dieser ,Meereshöhlen" betont bereits Th. Fuchs (1894, p. 54), und das insbesondere in Hinblick darauf, daß diese vom Licht abgeschlossenen Marinbiotope mit dem ebenfalls lichtlosen Lebensbereich ,Tiefsee“ ökologisch verwandt sind. Der genannte Autor weist auf den Blindfisch Stygicola dentata (Poey, 1856) hin, der auf Kuba in Höhlen vorkommt, ,, welche mit dem Meere kommuniciren", und dessen Nächstverwandte in der Tiefsee verbreitet sind, was sich insbesondere in der auffallenden Ähnlichkeit mit dem blinden Aphyonus gelatinosus zeigt, der bei Neu-Guinea in einer Tiefe von 1400 Faden erbeutet wurde.

Die gleichen ökologischen Beziehungen zwischen Marinhöhlen und Tiefsee belegt Th. Fuchs (1894, p. 55) mit dem Auffinden von Munidiopsis polymorpha Koelbel 1892 (Decapoda, Reptantia, Anomura), die in einer mit dem Meere in Verbindung stehenden Höhle im Untergrund der Insel Lanzarote lebt (vgl. K. Koelbel, 1892, p. 105) und die einer ,,ausgesprochenen Tiefseegattung" angehört, die ,,bisher nur in Tiefen von 100-2000 Faden gefunden" wurde.

Es ist bemerkenswert, daß Th. Fuchs (1894, p. 55) bereits den Hinweis gibt, daß es bekanntlich , auch im Gebiet des Mittelmeeres mit dem Meere kommunicirende und mit Meerwasser gefüllte Höhlen“" gibt und daß es ,, sicherlich von großem Interesse" sei, auch diese Höhlen zu untersuchen. Es mußte jedoch erst ein halbes Jahrhundert vergehen, bis es neuerdings den von beispielhaftem Unternehmungsgeist getragenen Forschungen der Arbeitsgruppe Riedl (R. Riedl, 1959, 1959 a, 1959 b, 1963, 1964, 1966 ; E. F. Abel, 1959, 1959 a; K. Banse, 1959; G. Czihak, 1954; K. Russ \& K. Rützler, 1959; F. Starmühlner, 1955, 1955 a; W. Wieser, 1954; sowie J. Laborel et J. Vacelet, 1958) zu verdanken ist, daß dieser Lebensraum der Meereshöhlen in seiner Vielfalt und ökologischen Sonderstellung intensiv erfaßt wurde.

Da es nun aber mit dem Meere in Verbindung stehende Höhlensysteme gibt, in denen landeinwärts der marine Einfluß nahezu abklingt, können limnische und marine Höhlenregionen mehr oder weniger kontinuierlich ineinander übergehen. Daher kann auch diese Studie über die ökologische Position der limnischen Höhlengewässer innerhalb des Ökoregnums „Stygon“ nicht abgeschlossen werden, ohne diesem ,,ökologischen Gefälle“ zwischen ,Limnotroglon“" (=, Troglostygon") und, Thalassotroglon" zu folgen. 
Der obenerwähnte, zugleich trennende und verbindende „Grenzunschärfebereich" (vgl. G. H. Schwabe u. H. Klinge, 1960) zwischen Limnotroglon und Thalassotroglon zeigt, wie eng die Berührungspunkte liegen, an denen ,Limnospeologie“ und ,Thalassospeologie“ zusammentreffen. Eine somit von beiden Forschungsrichtungen aus naheliegende Inbeziehungsetzung makrokavernaler limnischer und mariner Lebensbereiche würde jedoch nur ein unvollständiger Brükkenschlag zwischen mariner und limnischer Subterranökologie bleiben, wenn nicht die ebenfalls in ökologischen Grenzunschärfebereichen ineinander übergehenden mikrokavernalen limnischen und marinen Lebensstätten entsprechend in Beziehung gesetzt werden. Das soll hier nur andeutungsweise vorgenommen werden, indem unserem „Stygopsammal" das ,Thalassopsammal" und dem „Stygopsephal" ein ,Thalassopsephal" zur Seite gestellt wird (Abb. 5).

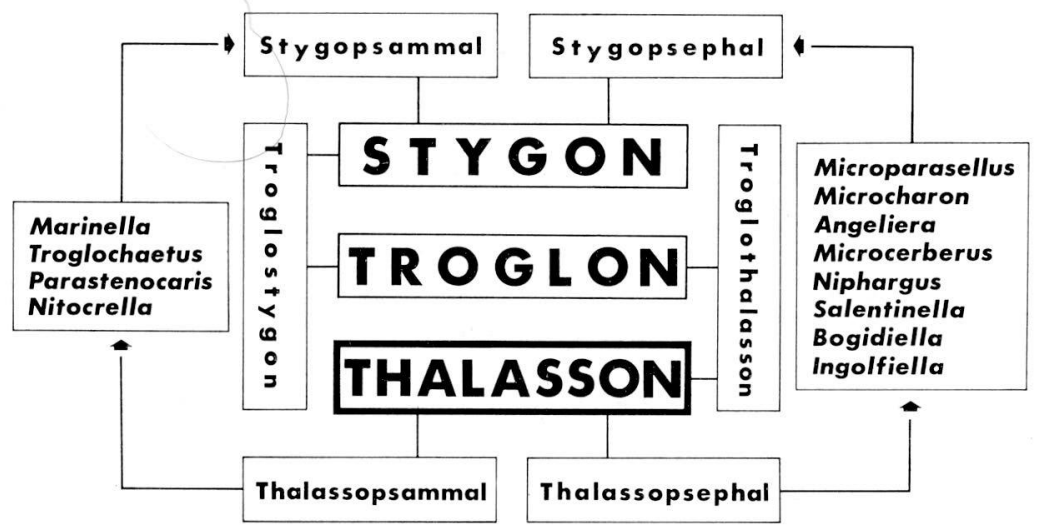

Abb. 5. Die ökologische und zönologische Verknüpfung mikro- und makrokavernaler Biotope und Biozönosen der Ökoregionen „Stygon“, ,,Thalasson" und , Troglon".

Wie eng gerade diese mikrokavernalen grundwasserführenden Biotope ökologisch verzahnt sind, geht daraus hervor, daß eine ganze Reihe limnischer Grundwassertiere im Mesopsammal beziehungsweise im Mesopsephal den ökologischen Übergang vom marinen zum limnischen Grundwasser vollziehen konnte (Abb. 5), und da einige dieser Immigranten sogar bis in limnische Höhlengewässer vorgedrungen sind (z. B.: Troglochaetus, Parastenocaris, Nitocrella, Microparasellus, Microcharon, Salentinella, Niphargus), wird ersichtlich, wie angebracht es ist, daß ,Limnospeologie“" und ,Thalassospeologie" sowie limnologische und marinökologische Grundwasserforschung ihr jeweiliges Arbeitsgebiet als Teil eines zusammengehörenden Ganzen sehen. 


\section{ZUSAMMENFASSUNG}

,,Höhlen-“ und „,Spaltengewässer" werden als subterranaquatile Ökosysteme (,,Troglostygon", ,,Petrostygon") den übrigen grundwasserführenden Lebensbereichen (z.B.,,Eustygon", ,,Stygorhithron“", ,Stygopotamon“) gegenübergestellt.

Die Besiedlung unterirdischer Biotope wird auf die Wirkung eines ,,Grundprinzips der Herausbildung von Hauptökotopen der Grundwasserfauna" zurückgeführt.

Nach dieser ökologischen Gesetzmäßigkeit, die im einzelnen erläutert und formuliert wird, sind grundwasserführende Lückenbiotope (z. B. ,,Eustygopsammal“", ,Rhithrostygopsephal“", ,Potamostygopsammal) als eigentliche Lebensbereiche der stygobionten Grundwasserfauna anzusehen, Höhlengewässer aber als Sekundärbiotope.

Die von Tieren besiedelten Meereshöhlen werden als Ökosystem ,,Thalassotroglon" in ihren Beziehungen zum ,Limnotroglon" (,,Troglostygon“) dargestellt.

Bei der damit vollzogenen Kontaktaufnahme zwischen „Limnospeologie" und ,Thalassospeologie" werden auch die limnischen und marinen mikrokavernalen Lebensbereiche mit berücksichtigt: ,"Thalassopsammal“ und ,,Thalassopsephal".

„Limnospeologie“ und „Thalassospeologie“ sowie limnologische und thalassologische Grundwasserforschung werden als biologische Arbeitsrichtungen charakterisiert, die der Erforschung eines ökologisch verknüpften Ganzen dienen.

\section{SUMMARY}

Bodies of waters in caves and in crevices of rocks are distinguished from the other subsoil water ecosystems ("eustygon", "stygorhithron", "stygopotamon") under the names "troglostygon" and "petrostygon".

The colonisation of subsoil water biotopes involves a fundamental principle which controls the development of the main biotopes for the stygobiont undergroundwater organisms.

According to this ecological rule, which is described in detail and formulated, the several interstitial biotopes (for example "eustygopsammal," "rhithrostygopsammal," "potamostygopsephal") are to be considered as the real biotopes of the stygobiont subsoil water organisms; waters in caves, on the contrary, are secondary biotopes of these animals.

Caves which contain marine water are described as ecostystem "Thalassotroglon" in their relation to "limnotroglon" (= "stygotroglon").

In this way the contact between "limnospeology" and "thalassospeology" is established, and the limnic and marine microcavernal biotopes - "thalassopsammal" and "thalassopsephal" - are also taken in consideration.

"Limnospeology" and "thalassospeology" as limnological and thalassological investigations of subsoil water are characterized as biological fields of work, which serve for the investigation of an ecological unit.

\section{LITERATUR}

Aвец, E. F. (1959) - Zur Kenntnis der marinen Höhlenfauna unter besonderer Berücksichtigung der Anthozoen. Ergebn. d. Österr. TyrrheniaExpedition 1952, Teil V. Pubbl. Staz. Zool. Napoli, Suppl. 30:1-94. 
AвeL, E.F. (1959 a) - Zur Kenntnis der Beziehungen der Fische zu Höhlen im Mittelmeer. Ergebn. d. Österr. Tyrrhenia-Expedition 1952, Teil XIV. Pubbl. Staz. Zool. Napoli, Suppl. 30: 519-527.

Angelier, E. (1953) - Recherches écologiques et biogéographiques sur la fauna des sables submergés. Arch. Zool. Expér. Gén. 90: 37-161.

BAnse, K. (1959) - Über die Polychaeten-Besiedlung einiger submariner Höhlen. Ergebn. d. Österr. Tyrrhenia-Expedition 1952, Teil XII. Pubbl. Staz. Zool. Napoli, Suppl. 30: 417-469.

Benedict, J. E. (1896) - Preliminary descriptions of a new genus and three new species of Crustaceans from an artesian well at San Marcos, Texas. Proc. U.S. Nat. Mus., vol. 18: 615-617.

Briegleb, W. (1962) - Zur Biologie und Ökologie des Grottenolms (Proteus anguinus Laur. 1768). Z. Morph. Ökol. Tiere 51: 271-334.

Calman, W. T. (1907) - On a Freshwater Decapod Crustacean collected by W. J. Burchell at Pará in 1829. Ann. Mag. nat. Hist. 19: 295-299.

Carranza, J. (1954) - Description del primer bagre anoftalmo y depigmentado en aguas Mexicanes. Ciencia XIV: 129-136.

Chace, F. A. (1943) - Two new blind Prawns from Cuba with a Synopsis of the subterranean Caridea of America. Proc. New Engl. zool. Cl. $22: 25-40$.

- (1954) - Two new subterranean shrimps (Decapoda: Caridea) from Florida and the West Indies, with a revised key to the American species. Journ. Wash. Acad. Sci. 44: 318-324.

Chace, F. A., und Holthuis, L. B. (1948) - Land and fresh water Decapod Crustacea from the Leerward Group and Northern South America. In: Hummelinck, Studies on the fauna of Curaçao, Aruba, Bonaire and the Venezuelan Islands 3: 21-28.

Chappurs, P. A. (1944) - Die Grundwasserfauna der Körös und des Szamos. A Magyar Tudományos Akadémia Kiadasa: 1-42.

- (1948) - Copépodes, Syncarides et Isopodes des eaux phréatiques de Suisse. Rev. Suisse Zool. 55 : 549-566.

- (1950) - La récolte de la faune souterraine. Notes biospéol. V: 7-35.

Craig-Bennett, A. (1938) - Record of Typhlocaris galilaea. Nature, 142 : 797.

Czıнак, G. (1954) - Die Schlangensterne einer submersen Höhle des Tyrrhenischen Meeres. Ergebn. d. Österr. Tyrrhenia-Expedition 1952, Teil III. Österr. Zool. Zeitschr. V: 15-29.

Defamare-Deboutteville, C. (1960) - Biologie des eaux souterraines littorales et continentales: 1-740. Herman, Paris.

Eigenmann, C. H. (1898) - The origin of cave faunas. Proceed. Ind. Acad. Sc. (1897): 229-230.

- (1905) - Divergence and Convergence in Fishes. Biol. Bull. VIII : 59-66.

- (1909) - Cave Vertebrates of America. A study of degenerative evolution. Carnegie Institut Washington Public. 104: 13.

- (1919) - Trogloglanis pattersoni, a new blind fish from San Antonio, Texas. Proceed. americ. philos. Soc. LVIII : 397-400.

Fuchs, Tн. (1894) - Über Tiefseetiere in Höhlen. Ann. K. K. Naturhist. Hofmuseum Wien IX: 54-55.

Garman, H. (1892) - The origin of the cave fauna of Kentucky, with a description of a new blind beetle. Science XX: 240-241. 
Gianferrari, L. (1923) - Uegitglanis zammaranoi, un nuevo siluride cieco africano. Atti. Soc. Ital. Sc. Nat. Milano LXII : 1-3.

Girard, M. D. (1859) - Ichthyological Notices. Proc. Acad. Nat. Sci. Phila.: 62-64.

Goeldi, E. (1904) - Nova zoologica aus der Amazonas-Region. Neue Wirbeltiere. Comptes-Rendus 6e Congrès internat. Zoologie, Berne: 542-549.

Hadzi, J. (1958) - Fortschritte in der Erforschung der Höhlenfauna des Dinarischen Karstes. Verh. Dtsch. Zool. Ges. (1957) 21:470-477.

- (1965) - Bemerkungen zu einigen biospeläologischen Problemen des Dinarischen Karstes. Nase Jame VII : 21-31.

Haine, E. (1946) - Die Fauna des Grundwassers von Bonn mit besonderer Berücksichtigung der Crustaceen.-Inaugural-Diss., Melle in Hannover.

Hertzog, L. (1936) - Crustaceen aus unterirdischen Biotopen des Rheintales bei Straßburg. I. Mitt. Zool. Anz. 114: 271-279.

Holthuis, L. B. (1956) - An enumeration of the Crustacea Decapoda Natantia inhabiting subterranean waters. Vie et Milieu VII : 43-76.

- (1960) - Two new species of atyid shrimps from subterranean waters of N.W. Australia (Decapoda Natantia). Crustaceana I : 47-57.

Huввs, C. L. (1936) - Fishes of the Yucatan Peninsula. Carnegie Institut Washington Public. 457: 157.

Huвbs, C. L., und Bailey, R. M. (1947) - Blind Catfishes from artersian waters of Texas. Occas. Papers Mus. Zool. Univ. Michigan 499.

Husman N, S. (1956) - Untersuchungen über die Grundwasserfauna zwischen Harz und Weser. Arch. Hydrobiol. 52: 1-184.

- (1957) - Die Besiedelung des Grundwassers im südlichen Niedersachsen. Beitr. Naturkde. Nieders. 10: 87-96.

- (1958) - Sand- und Schotterufer als Grenzbereiche limnologischer und bodenbiologischer Forschung. Gewässer und Abwässer 22: 66-69.

- (1966) - Versuch einer ökologischen Gliederung des interstitiellen Grundwassers in Lebensbereiche eigener Prägung. Arch. Hydrobiol. 62: 231-268.

ЈАковг, Н. (1954) - Biologie, Entwicklungsgeschichte und Systematik von Bathynella natans Vejd. Zool. Jb. Syst. 83:1-184.

Karaman, St. (1935) - Die Fauna der unterirdischen Gewässer Jugoslawiens. Verh. Int. Ver. Limnol. 7: 46-73.

- (1954) - Über unsere unterirdische Fauna. Acta. Mus. Maced. Sc. Nat. I: 195-216.

Kiefer, F. (1957) - Die Grundwasserfauna des Oberrheingebietes mit besonderer Berücksichtigung der Crustaceen. Beitr. nat. Forsch. Südwestdeutschland 16:65-91.

- (1960) - Psammobionte Ruderfußkrebse (Crust. Cop.) aus dem Gebiet der Unterweser und von der Insel Helgoland. Zool. Anz. 165: 30-37.

Koelbel, K. (1892) - Beiträge zur Kenntnis der Crustaceen der Canarischen Inseln. Ann. K. K. Naturhist. Hofmuseum Wien VII : 105-116.

Krause, E. (1898) - Die Augen der Blindfische. Promethius 457: 651-652.

KulHavý, V. (1961) - Über das Vorkommen der west- und osteuropäischen Elemente in der Crustaceenfauna der böhmischen unterirdischen Gewässer. Acta soc. zool. Bohemoslov. 25: 297-301.

Laborel, J. u. Vacelet, J. (1958) - Etude des peuplements d'une grotte sous-marine du golfe de Marseille. Bull. Inst. Océanogr. Monaco 55: $1-21$. 
Mees, G. F. (1962) - The Subterranean Freshwater Fauna of Yardie Creek Station, North West Cape, Western Australia. J. Roy. Soc. West. Austral. XLV: 24-32.

Menon, A. G. (1950) - On a remarkable blind Siluroid fish of the family Clariidae from Kerala (India). Rec. Indian Mus. XLVIII, I: 59-66.

MeEšrov, M. (1960) - Faunistisch-ökologische und biozönologische Untersuchungen unterirdischer Gewässer des Savetales. Bioloski Glasnik 13: 73-109.

Miers, E. J. (1877) - On a collection of Crustacea, Decapoda and Isopoda, chiefly from South America, with descriptions of new genera and species. Proc. zool. Soc. Lond. 1877: 653-679.

Мотаş, C. (1958) - Freatobiologia, o noua ramura a limnologiei : Natura 10 : 95-105.

- (1962) - Procédé des sondages phréatiques. - Division du domaine souterrain. - Classification écologique des animaux souterrains. - Le psammon. Acta. Mus. Mac. Sci. Nat. 8: 135-173.

Müller, G. (1931) - Sopra due Crostacei delle nostre acque carsiche. Atti. Mus. Stor. nat. Trieste 11: 206-216.

Myers, G. S. (1944) - Two extraordinary new blind Nematognath fishes from the Rio Negro representing a new subfamily, with a rearrangement of the genera of the family and illustrations of some previously described genera and species from Venezuela and Brazil. Proceed. California Acad. Sc. (4), XXIII, No. 40: 593-594.

NeEL, J. (1948) - A limnological investigation of the psammon in Douglas Lake. Trans. Amer. Microsc. Soc. 67: 1-53.

Noodt, W. (1952) - Subterrane Copepoden aus Norddeutschland. Zool. Anz. 148: 331-341.

Orghidan, T. (1955) - Un nou domeniu de viata acvatica subterana „,Biotopul Hiporeic". Bul. sti. sect. Biologie si sti. Agr. si sect. Geologie si Geografie 7: 657-676.

- (1959) - Ein neuer Lebensraum des unterirdischen Wassers: der hyporheische Biotop. Arch. Hydrobiol. 55: 392-414.

Pellegrin, J. (1929) - L'Eilichthys microphthalmus Pellegrin. Poisson cavernicole de la Somalie Italienne. Bull. Mus. Hist. Nat. Paris I: 363-364.

Pennak, R. W. (1940) - Ecology of the microscopic Metazoa inhabiting the sandy beaches of some Wisconsin lakes. Ecol. Monogr. 10: 537-615.

Pigard, J. Y. (1962) - Contribution a la connaissance de la faune psammique de Lorraine. Vie et Milieu 13: 471-505.

Priesel-Dichtr, G. (1959) - Die Grundwasserfauna im Salzburger Becken und im anschließenden Alpenvorland. Arch. Hydrobiol. 55: 281-370.

Regan, T. (1940) - The Fishes of the Gobiid genus Luciogobius Gill. Ann. Mag. nat. Hist. V: 462-465.

Remane, A. (1940) - Einführung in die zoologische Ökologie der Nord- und Ostsee. Tierw. Nord- u. Ostsee 1 a.

Riedi, R. (1959) - Die Hydroiden des Golfes von Neapel und ihr Anteil an der Fauna unterseeischer Höhlen. Ergebn. d. Österr. TyrrheniaExpedition 1952, Teil XVI. Pubbl. Staz. Zool. Napoli, Suppl. 30: $589-755$. 
RIE DEL, R. (1959 b) - Turbellarien aus submarinen Höhlen, 4. Faunistik und Ökologie. Ergebn. d. Österr. Tyrrhenia-Expedition 1952, Teil X. Pubbl. Staz. Zool. Napoli, Suppl. 30: 333-399.

- (1963) - Probleme und Methoden der Erforschung des litoralen Benthos. Verh. Dtsch. Zool. Ges. Wien 1963, Suppl. 26: 505-567.

- (1964) - Die Erscheinungen der Wasserbewegung und ihre Wirkung auf Sedentarier im mediterranen Felslitoral. Helgol. Wiss. Meeresunters. 10: $155-186$.

— (1966) - Biologie der Meereshöhlen. : 1 -636. P. Parey, Hamburg u. Berlin.

Russ, K., und Rützler, K. (1959) - Zur Kenntnis der Schwammfauna unterseeischer Höhlen. Ergebn. d. Österr. Tyrrhenia-Expedition 1952, Teil XVII. Pubbl. Staz. Zool. Napoli, Suppl. 30: 756-787.

Ruttaner-Kolisko (1957) - Der Lebensraum des Limnopsammals. Verh. Dtsch. Zool. Ges. Hamburg, Zool. Anz., Suppl. 20: 421-427.

Sassuchin, D. N., Kabanov, N. M., und Neizwestnova, K. S. (1927) - Über die mikroskopische Pflanzen- und Tierwelt der Sandfläche des Okaufers bei Murow. Russ. hydrobiol. Z. 6: 59-83.

Sch Ä fer, H. W. (1951) - Über die Besiedlung des Grundwassers. Verh. Internat. Verein. Limnol. 11: 324-330.

Sснмiтt, W. L. (1936) - Macruran and Anomuran Crustacea from Bonaire, Curaçao and Aruba. Zoologische Ergebnisse einer Reise nach Bonaire, Curaçao und Aruba im Jahre 1930. Zool. Jb. Syst. 67: 363-378.

Schwabe, G. H., und Klinge, H. (1960) - Gewässer und Boden als Forschungsgegenstand. Anales de Edafologia y Agrobiologia XIX: $519-568$.

Schwoerbel, J. (1961) - Über die Lebensbedingungen und die Besiedlung des hyporheischen Lebensraumes. Arch. Hydrobiol., Suppl. - FalkauSchriften - 25: 182-214.

- (1961 a) - Subterrane Wassermilben (Acari: Hydrachnellae, Porohalacaridae und Stygothrombiidae), ihre Ökologie und Bedeutung für die Abgrenzung eines aquatischen Lebensraumes zwischen Oberfläche und Grundwasser. Arch. Hydrobiol., Suppl. - Falkau-Schriften - 25: $242-306$.

- $(1964 b)$ - Die Bedeutung des Hyporheals für die benthische Lebensgemeinschaft der Fließgewässer. Verh. Internat. Verein. Limnol. 15: $215-226$.

Starmühlner, F. (1955) - Zur Molluskenfauna des Felslitorals und submariner Höhlen am Capo di Sorrento (Teil 1). Ergebn. d. Österr. Tyrrhenia-Expedition, Teil IV. Österr. Zool. Zeitschr. 6: 147-249.

- (1955a) - Zur Molluskenfauna des Felslitorals und submariner Höhlen am Capo di Sorrento (Teil 2). Ergebn. d. Österr. Tyrrhenia-Expedition, Teil IV. Österr. Zool. Zeitschr. 6: 631-713.

Teschner, D. (1963) - Die Biologie, Verbreitung und Ökologie der Grundwassermilbe Lobohalacarus weberi quadriporus (Walter 1947), Limnohalacaridae, Acari. Arch. Hydrobiol. 59: 71-102.

Thienemann, A. (1925) - Die Binnengewässer Mitteleuropas. Die Binnengewässer 1: 1-255. Schweizerbart, Stuttgart.

Thines, G. (1958) - Observations sur les habitats de l'Uegitglanis zammaranoi Gianferrari 1923, Clariidae aveugle de la Somalie italienne. Rev. Zool. Bot. Afric. LVII: 117-124. 
Uhlmann, D., und Schulze, E. (1961) - Zur Limnologie von Höhlengewässern. Verh. Int. Verein. Limnol. 14: 862-866.

VAndel, A. (1964) - Biospéologie. La Biologie des Animaux Cavernicoles: 1-619. Gauthier-Villars, Paris.

Wegelin, R. (1966) - Beitrag zur Kenntnis der Grundwasserfauna des Saale-Elbe-Einzugsgebietes. Zool. Jb. Syst. 93: 1-117.

Wieser, W. (1954) - Beiträge zur Kenntnis der Nematoden submariner Höhlen. Ergebn. d. Österr. Tyrrhenia-Expedition 1952, Teil II. Österr. Zool. Zeitschr. V: 172-230.

Woods, L. P., und Inger, R. F. (1957) - The Cave, Spring and Swamp Fishes of the Family Amblyopsidae of Central and Eastern United States. Amer. Midland Natural. LVIII : 231-256. 